\title{
Genome and transcriptome analysis to understand the role diversification of cytochrome P450 gene under excess nitrogen treatment
}

\author{
Rui Xiong ${ }^{1}$, Ting He${ }^{1}$, Yamei Wang ${ }^{1}$, Shifan Liu ${ }^{1}$, Yameng Gao ${ }^{2}$, Hanwei Yan ${ }^{1,2^{*}}$ and Yan Xiang ${ }^{1,2^{*}}$
}

\begin{abstract}
Background: Panax notoginseng (Burk.) F. H. Chen (P. notoginseng) is a medicinal plant. Cytochrome P450 (CYP450) monooxygenase superfamily is involved in the synthesis of a variety of plant hormones. Studies have shown that CYP450 is involved in the synthesis of saponins, which are the main medicinal component of P. notoginseng. To date, the $P$. notoginseng CYP450 family has not been systematically studied, and its gene functions remain unclear.

Results: In this study, a total of 188 PnCYP genes were identified, these genes were divided into 41 subfamilies and clustered into 9 clans. Moreover, we identified 40 paralogous pairs, of which only two had Ka/Ks ratio greater than 1 , demonstrating that most PnCYPs underwent purification selection during evolution. In chromosome mapping and gene replication analysis, 8 tandem duplication and 11 segmental duplication events demonstrated that PnCYP genes were continuously replicating during their evolution. Gene ontology (GO) analysis annotated the functions of 188 PnCYPs into 21 functional subclasses, suggesting the functional diversity of these gene families. Functional divergence analyzed the members of the three primitive branches of CYP51, CYP74 and CYP97 at the amino acid level, and found some critical amino acid sites. The expression pattern of PnCYP450 related to nitrogen treatment was studied using transcriptome sequencing data, 10 genes were significantly up-regulated and 37 genes were significantly down-regulated. Combined with transcriptome sequencing analysis, five potential functional genes were screened. Quantitative real-time PCR (qRT-PCR) indicated that these five genes were responded to methyl jasmonate (MEJA) and abscisic acid (ABA) treatment.
\end{abstract}

Conclusions: These results provide a valuable basis for comprehending the classification and biological functions of PnCYPs, and offer clues to study their biological functions in response to nitrogen treatment.

Keywords: Cytochrome P450, Panax notoginseng, Gene duplication, Expression profile, Nitrogen treatment

*Correspondence: hwyanahau@163.com; xiangyan@ahau.edu.cn; xiangyanahau@sina.com

${ }^{2}$ National Engineering Laboratory of Crop Stress Resistance Breeding,

Anhui Agricultural University, Hefei 230036, China

Full list of author information is available at the end of the article

\begin{abstract}
Background
Panax notoginseng (Burk.) F. H. Chen (P. notoginseng) belongs to the Araliaceae genus, and it is widely cultivated in Yunan province, China. P. notoginseng is a medicinal plant, and its medicinal records can be traced to 3000 years ago [1]. Previous researches have shown that $P$. notoginseng has numerous bioactive compounds, such as saponins, which are its the main medicinal ingredient [2]. A total of 56 dammarane-type saponins, such as
\end{abstract}

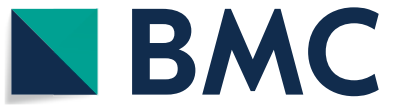

(c) The Author(s) 2021. Open Access This article is licensed under a Creative Commons Attribution 4.0 International License, which permits use, sharing, adaptation, distribution and reproduction in any medium or format, as long as you give appropriate credit to the original author(s) and the source, provide a link to the Creative Commons licence, and indicate if changes were made. The images or other third party material in this article are included in the article's Creative Commons licence, unless indicated otherwise in a credit line to the material. If material is not included in the article's Creative Commons licence and your intended use is not permitted by statutory regulation or exceeds the permitted use, you will need to obtain permission directly from the copyright holder. To view a copy of this licence, visit http://creativecommons.org/licenses/by/4.0/. The Creative Commons Public Domain Dedication waiver (http://creativeco $\mathrm{mmons}$.org/publicdomain/zero/1.0/) applies to the data made available in this article, unless otherwise stated in a credit line to the data. 
ginsenoside $\mathrm{Rg} 1, \mathrm{Rb} 1$ and notoginsenoside $\mathrm{R} 1$, have been identified and characterized [3]. These compounds can be used to treat cardiovascular diseases and trauma [1]; promote immunoregulation [4], hepatoprotection [5]; and anti-carcinogenesis [6]. No oleanane-type saponins were found in $P$. notoginseng. This was the main difference in chemical composition between $P$. notoginseng and other ginseng plants [2]. Significantly, the cytochrome P450 (CYP450) genes play roles in both saponin synthesis and plant hormone synthesis.

The CYP gene family is large and intricate, and is involved in the synthesis of various primary and secondary metabolites, such as phenylpropanes, terpenoids, alkaloids, fatty acids and hormone precursors [7]. CYP genes are widely found in animals, plants, fungi and bacteria [8]. Those genes are usually divided into 10 clans, including six single clans (clan 51, clan 74, clan 97, clan 710, clan 711, and clan 727) and four multiple gene family clans (clan 71 , clan 72 , clan 85 , and clan 86) [9]. The functions of some CYP450 subfamilies have been elucidated. CYP74B, CYP703 and CYP704 can catalyze the production of fatty acid hydroxylase [10-12]. CYP74A, which also known as allene oxide synthase (AOS), is a key enzyme in jasmonate (JA) synthesis [13]. CYP97 is involved in abscisic acid (ABA) synthesis and participates in lutein synthesis [14]. In addition, CYP707A, which is present as ABA 8'-hydroxylases (ABA8ox) can catalyze phaseic acid synthesis. In gibberellin (GA) production, ent-kaurene oxidase (KO, CYP701A) catalyzes ent-kaurenoic acid formation [15]. Ent-kaurenoic acid oxidase (KAO) (CYP88A) uses the acid as a substrate to produce the GA12-aldehyde [16]. CYP90B, CYP90C, CYP90D, CYP72, CYP85A, CYP734A and CYP724B are responsible for brassinosteroid (BR) biosynthesis [7]. CYP71 enzyme in Sorghum is known to participate in the biosynthesis of the benzoquinone allelochemical sorgoleone [17]. Furthermore, the two nicotine n-demethylase genes (CYP82E) destroy the formation of nicotine in tobacco [18]. Importantly, CYP450 is associated with saponin synthesis in some plants. Avena spp. CYP51H10 [19], Glycine max (L.) Merr. CYP93E1 [20], CYP88D6 and CYP72A154 of Glycyrrhiza uralensis Fisch [21, 22]., CYP716A12 in Medicago truncatula L. have been reported to be involved in the synthesis of saponin [23].

Apart from the function on metabolites synthesis, some CYP450 genes also play roles in resistance to stresses, such as drought, salt, pest infestation and bacterial pathogens. For instance, OsDSS1, which is a member of the cytochrome CY450 gene family, and its mutants (dwarf and small seed 1, dss1) enhance Oryza sativa drought tolerance due to the accumulation of ABA and metabolites [24]. The AtCYP709B3 T-DNA insertion mutants (cyp709b3) show a salt intolerance phenotype [25]. The transient expression of saponin synthesis pathway genes in tobacco leaves indicates that $B v C Y P 72 A 552$ catalyzed the formation of hederageninbased saponins [26]. The reaction products of hydroperoxide lyase (HPL, CYP74B) in Arabidopsis thaliana, can be involved in the deterrence of insect pests [27]. Oscyp71Z2 overexpression plants show obvious resistance to bacterial fusarium caused by rice-XOO [28]. Some CYP450 genes have a metabolic detoxification function, and are involved in the decomposition of environment toxins [29]. Under treatment with basic macronutrients such as nitrogen, the expression level of some genes of CYP450 can also change. Decreased transcript levels of PtCYP711A1 have been detected in roots with excess $\mathrm{N}$ treatment, and the transcript level of PtCYP707A1 is up-regulated in N-starved roots [30]. In addition to its functions on compounds synthesis and biological or abiotic stress resistance, CYP450 has also been reported to be a growth factor. The CYP78A mutation in Arabidopsis and rice indicate that CYP78A is involved in regulating organ size and cell proliferation [7].

In recent years, many articles focused on the $C Y P$ gene family systematical studies were published, including A. thaliana [31], O. sativa [32], Vitis vinifera [33] and Panax ginseng [34]. Whereas, identification, structural and evolutionary analysis of the $P n C Y P$ gene family have not been reported, and there are few studies on the function of $P n C Y P$ genes. In this study, we performedgenome-wide bioinformatics analysis of the $P n C Y P$ gene family, including phylogenetic analysis, gene structure, conserved motifs, chromosomal locations, gene duplications, cis-regulatory elements and functional divergence. Meanwhile, due to the high medicinal value of $P$. notoginseng, abusing nitrogen $(\mathrm{N})$ fertilizer is very common in $P$. notoginseng planting [35, 36]. Previous studies have shown that plant hormones combined with $\mathrm{N}$ signals regulate root growth and development [30, 37, 38], thereby the $P_{n} C Y P s$ expression pattern under nitrogen treatment have been studied. Genes related to the synthesis of plant hormones (ABA, MEJA, GA) and saponins were screened by transcriptomic data analysis, the tissue expression pattern and change trend of gene expression levels under hormone treatment were analyzed by quantitative realtime PCR (qRT-PCR). Furthermore, some studies have predicted that $C Y P$ genes may be the miRNA target $[32,39]$, so we utilized this prediction in our paper. All of these were helpful to study the CYPs function of $P$. notoginseng, and optimize the plant environment of $P$. notoginseng to cultivate excellent varieties. 


\section{Results}

\section{Classification of identified PnCYP450s}

The 188 putative CYP450 genes were obtained from $P$. notoginseng. Here, we designated the $P n C Y P$ genes according to the classification Of $p$. ginseng $C Y P$ genes by sequence similarity. The genes were classified into 9 clans with 40 subfamilies. They were grouped into A-type (CYP71 clan) with 17 subfamilies comprising 102 genes and non-A type (CYP51, 72, 74, 85, 86, 97, 710, 711 clans) with 18 subfamilies including 86 PnCYPs. The CYP92, CYP703A, CYP710A, CYP711A family contained only one gene, while the CYP71 family was the largest family containing 27 memebers. The sequence length of 188 CYP450 genes ranged from 618 to $6279 \mathrm{bp}$ and the molecular weights $(\mathrm{Mw})$ of these proteins ranged from 22.5 to $239 \mathrm{kDa}$. The isoelectric points ranged from 5.17 to 9.51 (Table S1).

\section{Phylogenetic analyses and selective pressure analyses of PnCYP genes}

An unrooted neighbor-joining (NJ) phylogenetic tree of $188 P$. notoginseng genes was built to explore the relationships among $P n C Y P$ genes. Those genes were classified into nine clans, some clans (CYP51, CYP74, CYP97, CYP710, CYP711) contained only one gene family, while the others (CYP71, CYP72, CYP85, CYP86) were multifamily clans (Fig. 1). CYP71 was the largest CYP450 clan with functional diversity. In $P$. notoginseng, the members of this branch accounted for $54.3 \%$ of the total. CYP71 clan members in other plants have been reported to play roles in plant secondary metabolism, such as amino acid derivatives, fatty acids, alkaloids, terpenoids and precursors of hormones [9].

To investigate the evolutionary relationships of plant CYP450s, an unrooted NJ tree among $P$. notoginseng, $A$. thaliana, P. ginseng, O. sativa, P. trichocarpa and P. patens was constructed (Fig. S1). A total of 47 subfamilies were identified in Arabidopsis, of which seven subfamilies (CYP73, CYP83, CYP93, CYP702, CYP705, CYP708, CYP709) were not found in P. notoginseng. Forty-one subfamilies were identified in $P$. ginseng, two subfamilies (CYP720 and CYP724) were not found in P. notoginseng, while CYP710 was not found in ginseng. Some families were only found in moss, including CYP752, CYP753, CYP751, CYP762 and CYP764 families. Meanwhile, CYP99 family, CYP723 family and CYP729 family were only present in rice. Moreover, O. sativa contained the CYP51H and CYP51G subfamilies, and other species only contained the CYP51G subfamily. A. thaliana, P. notoginseng, $P$. ginseng contained only the CYP74A and CYP74B subfamilies, but the other three plants contained at least three CYP74 subfamilies (e.g. CYP74C, CYP74D et al.).
To further explore the evolutionary patterns of the CYP450 gene family in plants, paralogs of $P$. notoginseng, orthologs betweeen $P$. notoginseng and $A$. thaliana, $P$. notoginseng and $P$. ginseng were identified and listed in Table S2. Forty paralog pairs in Pn-Pn, 26 ortholog pairs in Pn-At, 70 ortholog pairs in Pn-Pg were identified (Table S2). To investigate the selection pressure of the PnCYP450 genes, the non-synonymous substitution rate/ synonymous substitution rate $(\mathrm{Ka} / \mathrm{Ks})$ value of these homologous pairs was calculated (Table S3 and Table S4). In total, only two paralog pairs (PnCYP736A2/ PnCYP736A12, PnCYP716A2/PnCYP716A7) in Pn-Pn had $\mathrm{Ka} / \mathrm{Ks}$ ratios greater than 1 . Six orthologs pairs (PnCYP76A1/PgCYP76A2v2, $\quad P n C Y P 81 B 2 / P g C Y$ P81D3p, PnCYP72A4/PgCYP72A8, PnCYP74B1/ PgCYP74B1, PnCYP707A1/PgCYP707A3, PnCYP704C2/ PgCYP704C4) had Ka/Ks ratios larger than 1 (Fig. S2). All $\mathrm{Ka} / \mathrm{Ks}$ values of $P n-A t$ orthologs pairs were less than 1 . This indicated that most genes have undergone purification selection.

\section{Gene structure and duplication analyses}

The conserved motifs of CYP450 proteins in P. notoginseng were searched (Fig. 2 and S3). Some motifs were presented in most proteins, such as the K-helix (motif 2, EXXR), and motif 6 (proton-transfer groove (PERF)). Motifs 4 and 8 existed in almost all families except the CYP74 clan. While some motifs were only presented in specific families, such as motifs 12,16 and 17 in the CYP71 clan, motif 20 in CYP86 clan. Obviously, members of CYP51 clan and CYP74 clan appeared to lose some motifs. The motif 11-7-12-5-18-14-17-15-4-82-3-9-6-1-10-13 layout was generally conserved in the CYP71 clan, motif 1 (CXG) and motif 4 (AGXD/ET) were related to functional definition. The motif layout (11-20-5-14-19-4-8-2-9-6-1) was universally conserved in CYP86 clan. Motif 11-4-8-2-10 layout in CYP51 clan and motif 11-7-4-8-2-3-6-10 layout in the CYP85 clan.

We analyzed the gene structure of the $P_{n} C Y P$ genes (Fig. S3). Some subfamilies consisted of intronless genes, such as CYP77 and CYP710. In 71 clan, most members ( 83 genes, $81.4 \%$ ) contained only one intron, while all CYP701 subfamily members contained six introns. The characteristic of 72 clan was that most genes had 4 introns. In 86 clan, almost all subfamilies (CYP86, CYP94 and CYP96) members had only one intron except the CYP704 subfamily. In contrast, CYP97 clan and CYP85 clan had very complex gene structures, $P n C Y P 97 A 1$ contained the maximum number of 15 introns.

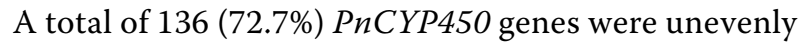
localized on the 12 chromosomes (Fig. 3). Chromosome 3 had the least genes at three, and chromosome 


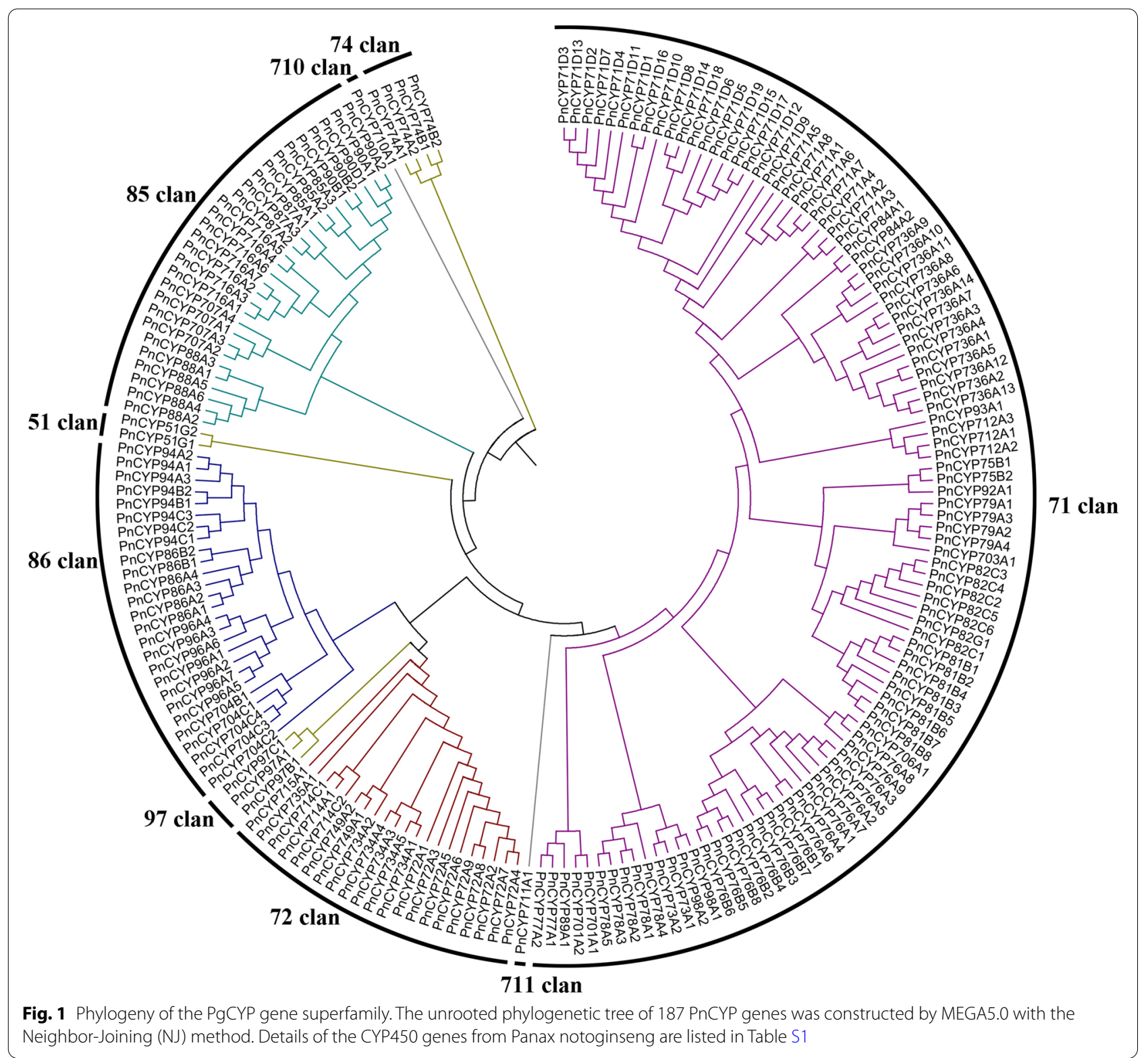

4 contained the most genes (23 total). Relatively higher gene density was observed in chromosome 4, 5, 6 and 10 regions. We defined 15 gene clusters (labelled a-o), the largest cluster (cluster e) which composed of 6 members was found on chromosome 5, and 8 paralogous pairs were found and thought to be evolved in tandem duplication (PnCYP71A2/PnCYP71A2, PnCYP72A4/PnCYP72A7, PnCYP71D8/PnCYP71D14, $P n C Y P 736 A 2 / P n C Y P 736 A 12$, PnCYP76A3/PnCYP76A5, PnCYP71D15/PnCYP71D17, PnCYP71D1/PnCYP71D16, PnCYP96A5/PnCYP96A7). And 11 segmental duplication events were detected in total.

\section{Cis-regulatory elements analyses and miRNA target prediction}

The 136 gene promoters contained two kinds of cis-regulatory elements (Fig. 4, Table S5), one of which was related to biological stress. Among these cis-regulatory regulatory elements, ABA response element (ABRE) accounted for the maximum ratio, 87 gene promoters (64\%) contained this element. The cis-regulatory elements associated with MEJA (CGTCA-motif and TGACG-motif) were respectively found in 72 and 73 gene promoters. There were another two cis-regulatory elements with a higher proportion: TGA-element (IAA-related, 53 genes) and TCA-element (salicylic acid (SA)-related, 58 genes). 


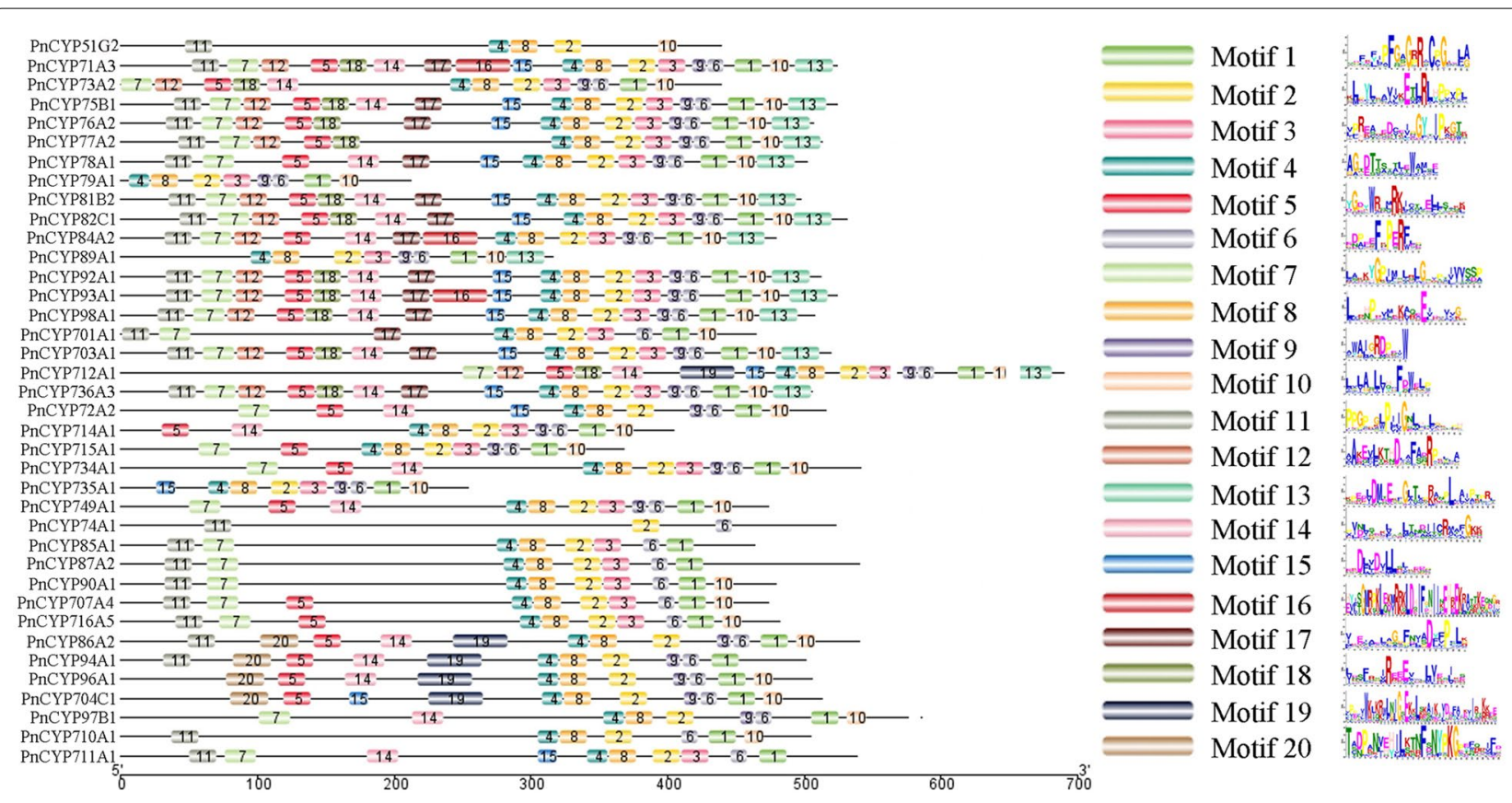

Fig. 2 The conserved motifs of PnCYP genes and their distribution in the genes. Conserved motifs in PnCYP genes were identified using MEME. Different colored boxes represent different motifs. Box lengths in the figure do not represent actual relative motif sizes

The promoters related to biological stress also included the GARE-motif and P-box elements which are involved in GA response. The other cis-regulatory element was related to abiotic stress, including MBS (41 genes) and G-box (94 genes) elements related to drought, low temperature stress response (LTR, 43 genes) and TC-rich (31 genes) repeats related to defense.

Three pairs of miRNA-target interactions were found, containing 2 miRNAs and 3 CYP450, (Table S6). MiR164 mediated transcriptional regulation of PnCYP736A9 and PnCYP736A10, while PnCYP86A3 was possibly a target of miR171. Both MiR164 and miR171 are associated with stress resistance in other species [40], while PnCYP736A9 and PnCYP86A3 were responded to nitrogen treatment in this study.

\section{GO, Kyoto encyclopedia of genomes (KEGG) annotation and subcellular localization prediction of the PnCYP450 gene superfamily}

A total of 187 PnCYP450s were annotated on Blast2GO software except PnCYP715A1. Those CYP450 protein were assigned to $21 \mathrm{GO}$ terms (Fig. 5a and Table S7). The terms were classified into three major functional categories, including 11 biological process subcategories, 7 cellular component subcategories and 3 molecular function subcategories. A total of 174, 176, and 186 proteins were annotated on the terms of biological process, cellular component, and biological process, respectively. Most genes were associated with metabolic reactions under biological process (GO:0008152), indicating that CYP450 genes function in the synthesis of various primary and secondary metabolites. Only three genes (PnCYP703A1, PnCYP90A2, PnCYP90A1) were predicted to be involved in cellular component organization or biogenesis (GO:0071840, GO:0022414, GO:0000003). For cellular component, most genes were related to membrane part (GO:0016020, GO:0044425) and only three genes (PnCYP94A1, PnCYP94A2, PnCYP86B1) were related to macromolecular complex (GO:0032991). On the terms of molecular function, most genes were shown to play roles in catalytic process (GO:0003824) but only one gene (PnCYP94A3) was related to the molecular function regulator.

A total of 77 PnCYP450s were annotated in the KEGG database. These genes were annotated on 17 pathways which were most related to compound synthesis (Fig. 5b). PnCYP73A1 and PnCYP73A2 were annotated into degradation of aromatic compounds (ko01220). PnCYP74 subfamily was related to alpha-Linolenic acid metabolism (ko00592). PnCYP82, PnCYP88, PnCYP701 subfamilies were shown to play roles in diterpenoid biosynthesis (ko00904). And PnCYP97, PnCYP707 subfamilies were predicted to have a function on carotenoid biosynthesis (ko00906). This indicates that CYP450 genes from different subfamilies may play roles in the same metabolic pathway. 


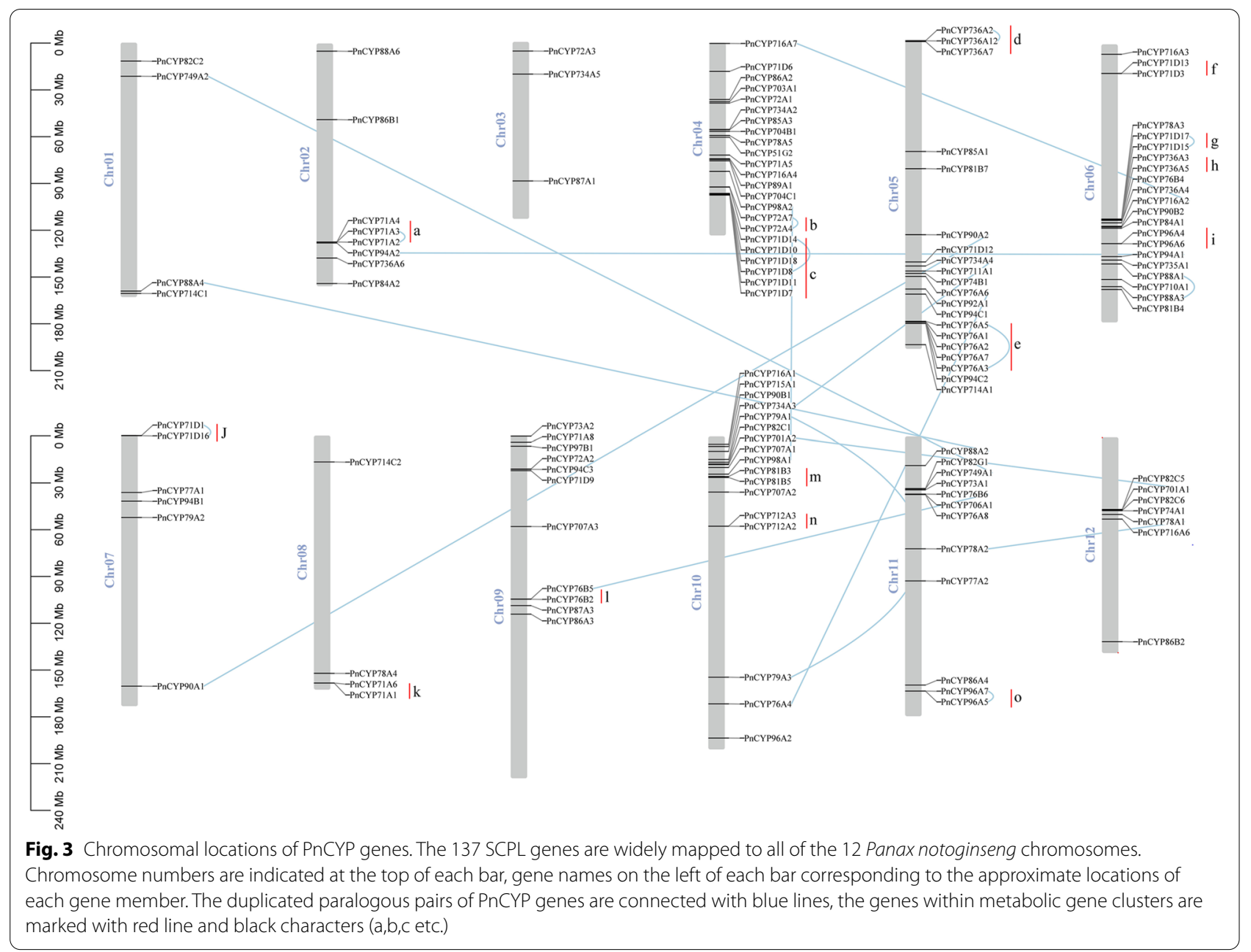

The subcellular localization analysis of the 188 PebHLH proteins predicted they were mostly located in the cytoplasmic (114, 60.6\%), 65 members were located in innermembrance $(34.6 \%)$, six were located in outermembrance (3.2\%), only three were located in periplasmic (1.6\%) (Table S7).

\section{Functional divergence analysis of the CYP51, CYP74 and CYP97 clans}

We calculated the Type I and Type II functional divergence correlation coefficient of CYP51, CYP74, and CYP97 clans, because these clans were conserved throughout evolution and had different functions [39]. As shown in Table 1 and S8, those three families were divided into five clusters: CYP51H/CYP51G, CYP74A/ CYP74B, CYP97A/CYP97B, CYP97A/CYP97C, CYP97B/CYP97C. In CYP51H/CYP51G pairs, Type I functional divergence coefficient was significantly greater than $0\left(\theta_{\mathrm{I}}, 0.4729 \pm 0.135917,{ }^{*} P<0.05\right)$, a total of 14 critical amino acid sites (CAASs) were detected. But the Type-II functional divergence coefficients $\left(\theta_{\mathrm{II}}\right)$ of this pair was less than 0 . A similar situation was also found in CYP74A/CYP74B pairs, the Type I functional divergence coefficient was statistically significant $\left(\theta_{\mathrm{I}}, 0.2984 \pm 0.091536,{ }^{*} P<0.05\right)$ and contained six CAASs. However, Type-II functional divergence coefficient was negative. These findings indicated that evolution rate divergence and evolution property divergence of CYP51, CYP74 clan were inconsistent. Meanwhile, the Type-I and Type-II coefficients of CYP97A/CYP97B pair $\left(\theta_{\mathrm{I}}, 0.5808 \pm 0.187075\right.$, $\left.{ }^{*} P<0.05 ; \theta_{\mathrm{II}}, 0.2443 \pm 0.058375, * P<0.05\right)$, CYP97A/ CYP97C pairs $\left(\theta_{\mathrm{I}}, 0.5336 \pm 0.161246,{ }^{*} P<0.05 ; \theta_{\mathrm{II}}\right.$, $\left.0.2374 \pm 0.055073,{ }^{*} P<0.05\right), C Y P 97 \mathrm{~B} / \mathrm{CYP} 97 \mathrm{C}$ pairs $\left(\theta_{\mathrm{I}}, 0.4912 \pm 0.150662,{ }^{*} P<0.05 ; \theta_{\mathrm{II}}, 0.2409 \pm 0.058073\right.$, * $P<0.05)$ were all significant. In Type I fuctional divergence, there were 29, 24, 17 CAASs in CYP97A/ CYP97B, CYP97A/CYP97C, CYP97B/CYP97C clusters, respectively. While in Type-II functional divergence, 

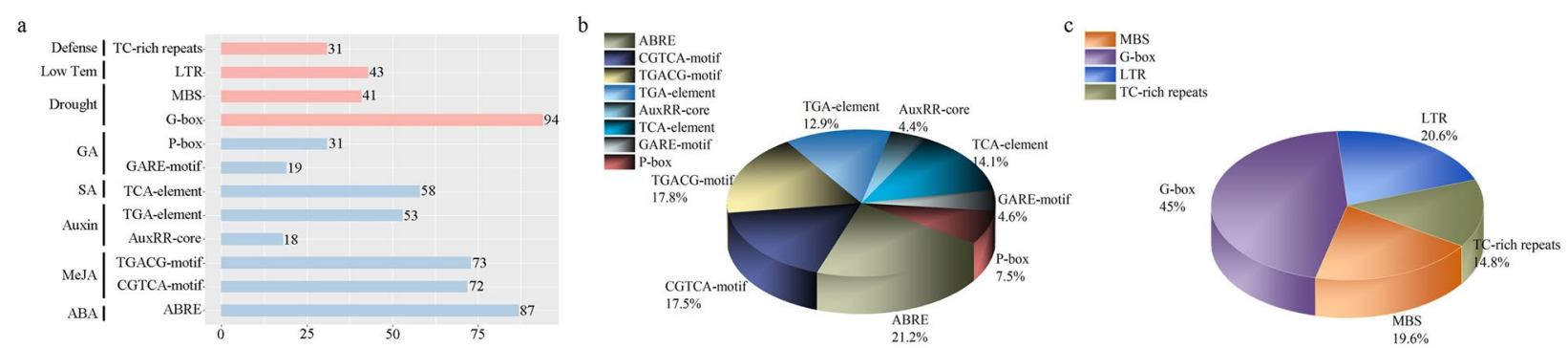

Fig. 4 Cis-acting element analysis of the promoter regions of PnCYP genes. Based on functional annotation data, cis-acting elements were classified into two major classes: phytohormone responsive elements (i.e. those responsive to ABA, auxin, GA, MeJA, and/or SA) and abiotic stress response cis-acting elements (e.g. those involved in plant defense, drought stress response, and/or low temperature stress response). a Number of each cis-acting elements in the promoter regions of PnCYP genes. $\mathbf{b}$ Percentage of total cis-acting elements in the promoter region of the PnCYP gene. $\mathbf{c}$ and $\mathbf{d}$ The number of each cis-acting element as a percentage of its classification
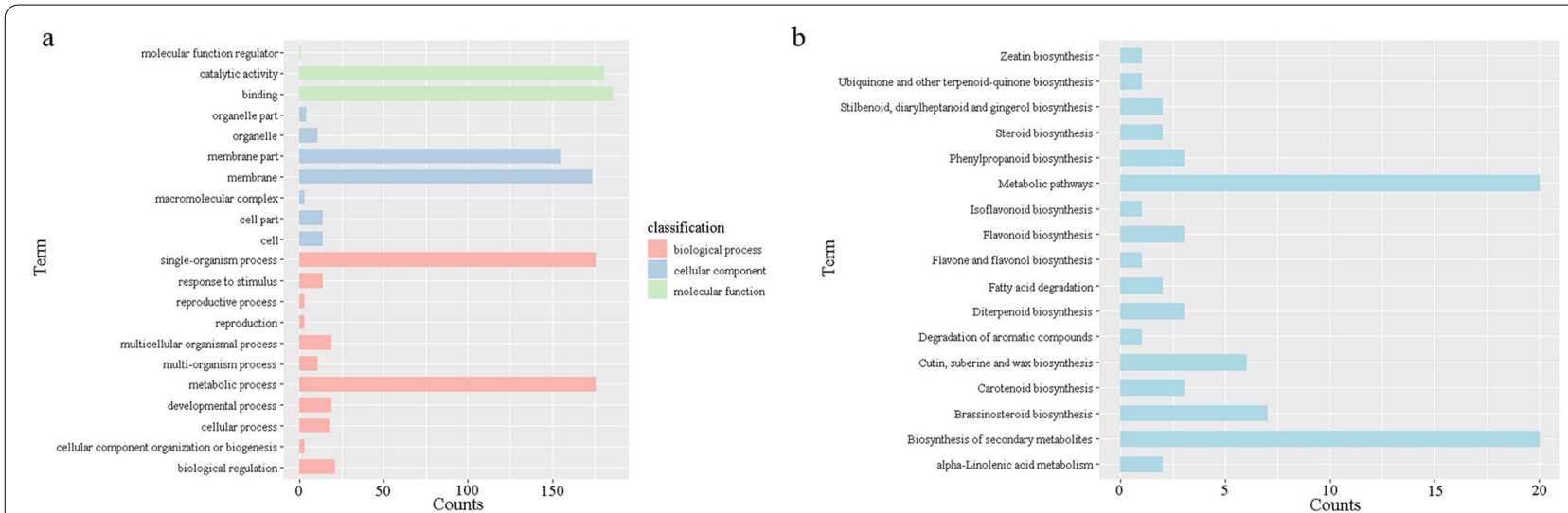

Fig. 5 Annotation analysis of PnCYP proteins. a: Gene ontology (GO) annotation of PnCYP proteins. Bars indicate the number of genes with the same term. b:Kyoto Encyclopedia of Genes and Genomes (KEGG) annotation of PnCYP proteins

Table 1 Site-specific profiles for two types of functional divergence (Type-I and Type-II), measured by the posterior ratio

\begin{tabular}{|c|c|c|c|c|c|c|}
\hline Comparison pairwise & Types & $\theta$ & LRT & $p$-value & $\begin{array}{l}\text { Cutoff of posterior } \\
\text { probability }\end{array}$ & $\begin{array}{l}\text { Number } \\
\text { of RFD }\end{array}$ \\
\hline CYP51H/CYP51G & । & $0.4729 \pm 0.135917$ & 12.105816 & $<0.01$ & 0.694309 & 14 \\
\hline CYP74A/CYP74B & । & $0.2984 \pm 0.091536$ & 10.627121 & $<0.01$ & 0.671592 & 6 \\
\hline CYP97A/CYP97B & । & $0.5808 \pm 0.187075$ & 9.638727 & $<0.01$ & 0.684058 & 29 \\
\hline CYP97A/CYP97C & । & $0.5336 \pm 0.161246$ & 10.951024 & $<0.01$ & 0.673728 & 24 \\
\hline CYP97B/CYP97C & I & $0.4912 \pm 0.150662$ & 10.629417 & $<0.01$ & 0.701093 & 17 \\
\hline CYP97A/CYP97B & $\|$ & $0.2443 \pm 0.058375$ & & $<0.01$ & 1.126562 & 52 \\
\hline CYP97A/CYP97C & $\|$ & $0.2374 \pm 0.055073$ & & $<0.01$ & 1.076256 & 62 \\
\hline CYP97B/CYP97C & $\|$ & $0.2409 \pm 0.058073$ & & $<0.01$ & 1.151247 & 69 \\
\hline
\end{tabular}

$\theta$ : the coefficients of Type-I and Type-II functional divergence between two gene clusters; LRT Likelihood ratio statistic Cutoff of posterior probability: minimum posterior probability of amino acid sites leading to functional divergence Number of RFD: the predicted number of amino acid sites associated with functional divergence 
52, 62 and 69 CAAs were detected in in CYP97A/ CYP97B, CYP97A/CYP97C, CYP97B/CYP97C clusters, respectively.

\section{Expression analyses of PnCYP450 genes treated with different forms of nitrogen fertilizers}

Excessive use of ammonium salt as the sole source of nitrogen hindered plant growth. To analyze expression levels of CYP450s in response to nitrogen fertilizers treatment, 188 PnCYP genes expression data (Per Kilobase of exon model per Million mapped reads, FPKM) were counted (Table S9). One hundred seven PnCYP genes whose expression data $>1$ in one or more treatment were selected for further analysis (Fig. S4, Table S10). A total of 47 differentially expressed genes were identified, among them, 10 genes were significantly up-regulated and 37 genes were significantly down-regulated (fold change $(\mathrm{FC})>1.5$ and $p$-values $<0.05)$ (Fig. 6, Table S11). Five genes (PnCYP71D6, PnCYP71D9, PnCYP71D16, PnCYP76B5, PnCYP736A7) were significant up-regulated under $15 \mathrm{~A}$ and $15 \mathrm{~N}$ treatment, however, when the plants were treated with $15 \mathrm{AN}$, the expression levels of these genes did not change significantly. The expression values of four genes (PnCYP71D18, PnCYP71D19, PnCYP84A2, PnCYP88A2) were significantly increased under the three treatments. While the expression levels of PnCYP93A2, PnCYP704C3, PnCYP716A6, PnCYP86B2, PnCYP93A4, PnCYP734A6, PnCYP78A2,

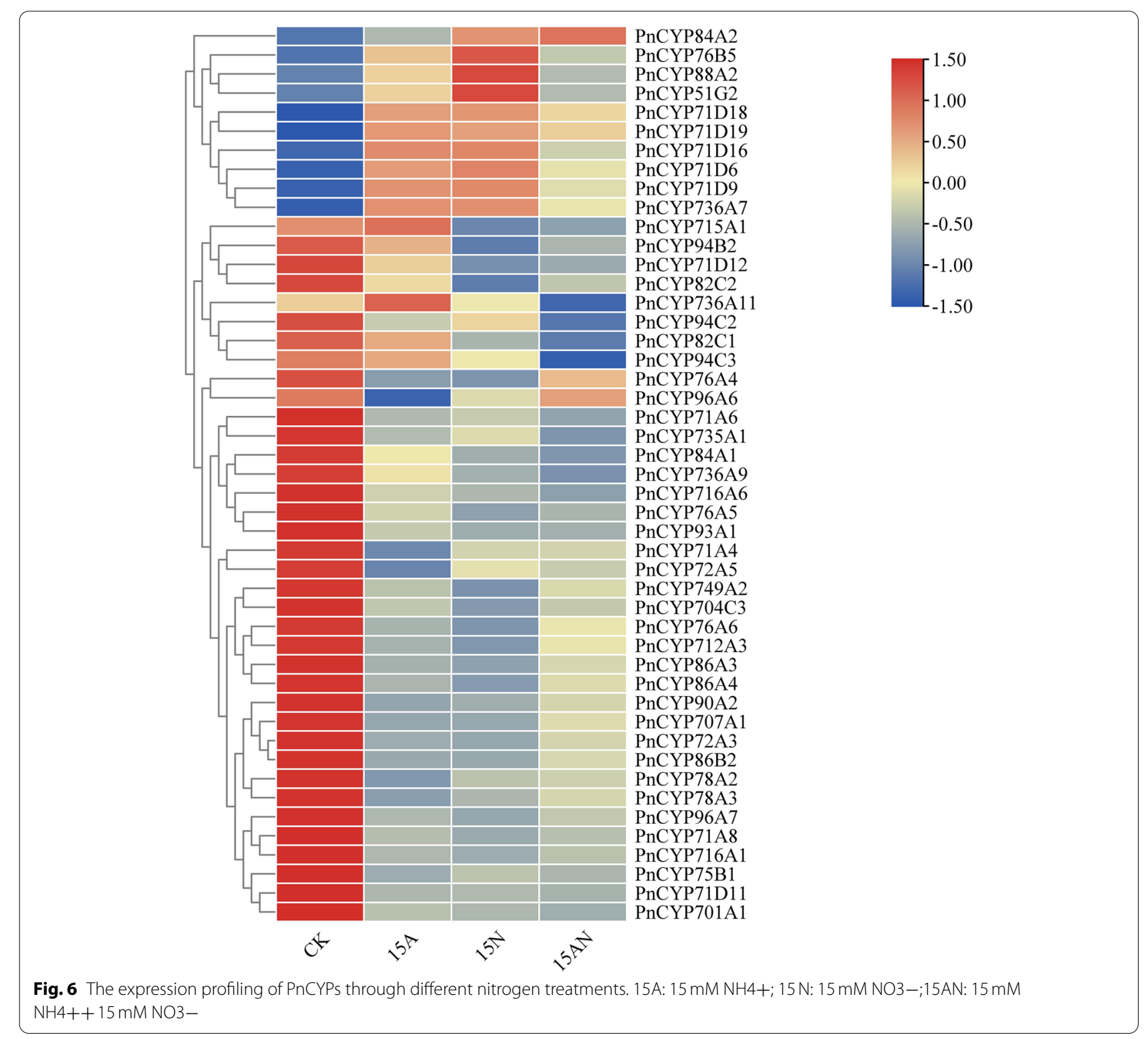


PnCYP76A5, PnCYP71A6, PnCYP96A7, PnCYP72A3, PnCYP75B1, PnCYP90A2, PnCYP71A4, PnCYP701A1, PnCYP707A1, PnCYP716A1, PnCYP71A8, PnCYP71D11 and $\mathrm{PnCYP78A} 3$ were reduced under all three treatments. PnCYP735A2, PnCYP82C1, PnCYP736A9, PnCYP71D12, PnCYP94B2 and PnCYP84A1 exhibited decreased expression under $15 \mathrm{~N}$ and $15 \mathrm{AN}$ treatments. PnCYP76A4, PnCYP76A6 and PnCYP86A4 responded to $15 \mathrm{~A}$ and $15 \mathrm{~N}$ treatment. PnCYP94C3, PnCYP94C2, PnCYP735A5, PnCYP86A3, PnCYP72A5 and PnCYP96A6 only responded to one treatment.

\section{Quantitative real-time PCR (qRT-PCR) expression profiles of PnCYP genes}

Nitrogen treatment can affect the synthesis of some hormones (ABA, MEJA, GA) in plants. In the present study, a MEJA synthesis related gene (PnCYP94B2), one gene related to GA synthesis (PnCYP701A1) and one gene related to ABA synthesis (PnCYP707A1) were screened for the further study. The main medicinal components in P. notoginseng were saponins. Therefore, two saponin synthesis related genes (PnCYP716A1, PnCYP716A6) were screened. Primers used for the
qRT-PCR analysis of PnCYP gene expression were listed in Table S13. Tissue expression pattern analysis showed that the expression levels of PnCYP94B2, PnCYP701A1 and PnCYP707A1 in leaves were higher than these in the other three tissues, while the expression levels of PnCYP716A1 and PnCYP716A6 in the roots were higher than these in the other three tissues (Fig. 7). We also analyzed the expression trends of these five candidate genes under $\mathrm{ABA}$ and MEJA treatment. The expression level of the PnCYP707A1 gene was significantly decreased under treatment with ABA and MEJA (>2), while the expression levels of the remaining four genes were all increased under treatment with these two hormones. In the MEJA treatment, PnCYP94B2, PnCYP701A1, PnCYP716A1 and PnCYP716A6 showed similar changes, that is, the expression levels of PnCYP94B2, PnCYP701A1, PnCYP716A6 were significantly increased at $1 \mathrm{~h}, 6 \mathrm{~h}$ and $12 \mathrm{~h}(>2)$. The expression patterns of PnCYP94B2 and PnCYP701A1 were similar in the ABA treatment, and the expression levels of PnCYP716A1 and PnCYP716A6 were significantly increased at $1 \mathrm{~h}, 3 \mathrm{~h}$, $6 \mathrm{~h}, 12 \mathrm{~h}$ and $24 \mathrm{~h}$ (Fig. 7 ).

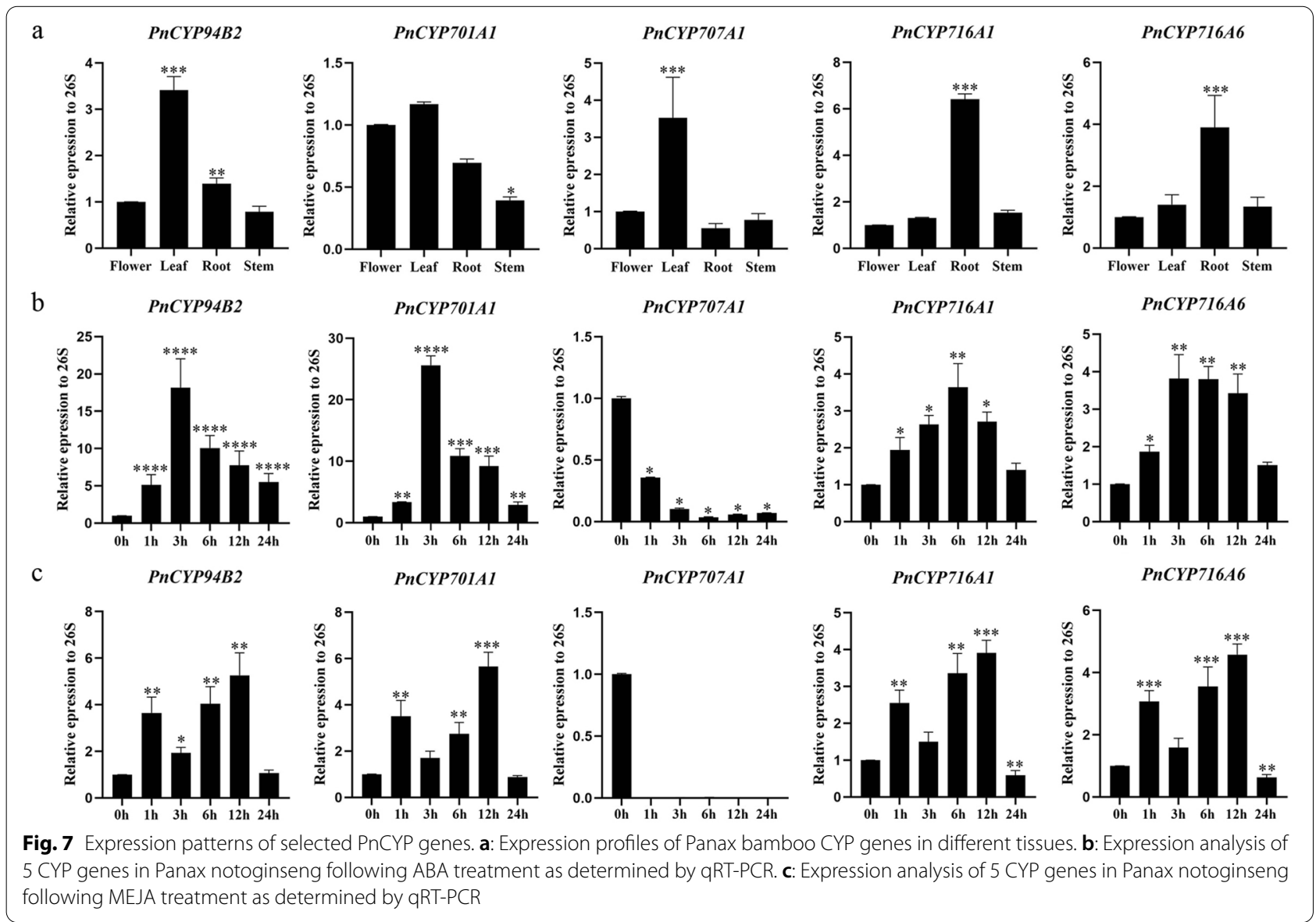




\section{Discussion}

To investigate the evolutionary relationships of plant CYP450s, an unrooted NJ tree among $P$. notoginseng, $P$. ginseng, Physcomitrella patens (moss), O. sativa (rice), A. thaliana (Arabidopsis), Populus trichocarpa (poplar) was constructed (Fig. S1). The number of PnCYPs was less than that of Arabidopsis (245), poplar (310), rice (326), and more than algae (98) and ginseng (138) [31-34], indicating that the CYP family has a significant degree of evolutionary extension among plant species. The molecular weight (MW) and isoelectric point (PI) partly determined the gene molecular structure and biochemical function [41], and the large range of PnCYP MW and PI may be due to their role in different synthetic pathways, which also shows the functional diversity of PnCYP. Protein divergence analysis showed that even in the conservative subfamilies, functional divergence still existed. In 51, 74, and 97 subfamilies, the type I functional divergence coefficients $\left(\theta_{\mathrm{I}}\right)$ among all branches were greater than 0 , indicating that functional divergence occurred between members of each subfamily. In addition, in the 97 subfamily, the $\theta_{\text {I }}$ coefficient was greater than that in the other two subfamilies, which proved that the functional divergence possibility in this subfamily was greater than that of 51,74 subfamily. Identifying potential critical amino acid positions in CYP51, CYP74, and CYP97 proteins can provide more information for analyzing the evolution of the PnCYP450 family.

Some subfamilies and clans showed closer evolutionary relationship. For example, CYP83 and CYP99 family were inside the CYP71 family on the phylogenetic tree. The monocot specific CYP723 family was obviously closest to CYP89 family, CYP729 family was obviously closest to CYP88 family. CYP51 clan, CYP710 clan and CYP85 clan were gathered on the same branch. Some members of the three clans were reported to have function on sterol synthesis and processing. CYP51G is reported to be involved in the synthesis of sterols, some CYP710 family members are known as sterol C22-desaturases, CYP85 clan members can process plant sterols [42]. In summary, CYP710 clan and CYP85 clan may be evolved from CYP51. Meanwhile, different subfamilies in the CYP85 clan (CYP90 and CYP724B subfamilies) are involved in the synthesis of brassinosteroids [43, 44], and phylogenetic tree analysis showed that the two subfamilies were clustered together. CYP74, CYP727 and CYP751 were also clustered on the same branch. CYP751 was only presented in moss and was clustered on the clan of CYP727, so we predicted that 751 and 727 may come from the same ancestor. CYP97, CYP72 and CYP86 clustering together on the phylogenetic tree indicated that they may be evolved from a common ancestor. CYP97 contained three distinct subfamilies, and CYP97C was scattered in the CYP97B branch. This proved that in the evolutionary process, the genetic relationship between CYP97B and CYP97C was closer, compared with CYP97A. The members of CYP72 clan are relevant to the synthesis of plant hormones (GA), cytokinins, isoprenoids and fatty acids $[11,44]$. Some members of CYP86 clan (CYP86 and CYP94 subfamilies) encode fatty acid hydroxylases or alkane hydroxylase $[45,46]$, and the two subfamilies were clustered together. Based on the above analysis, we speculated that the CYPS superfamily acquired new functions through duplicate divergence.

Motif and gene structure analysis strongly supported the results of subfamily classification. For some subfamilies in the same branch, motif compositions and gene structure were similar, such as the CYP71, CYP736 and CYP82 subfamilies in 71clan, indicating that these subfamilies might have similar functions. In this study, we found five cis-acting regulatory elements (ABA, MeJA, Auxin, SA and GA) associated with plant hormones were. The promoters of $P n C Y P 82 C 1, P n C Y P 701 A 1$, $P n C Y P 736 A 3$ and $P n C Y P 714 A 1$ contained all of these five cis-acting elements. At the same time, three cis-acting elements (Drought, Low Tem, Defense) associated with abiotic stress were found in the promoters. PnCYP76A5, PnCYP76A6, PnCYP78A5 and PnCYP72A2 contained all of these three cis-acting elements. This suggests that these genes may play an important role in the development of $P$. notoginseng. The expression patterns under nitrogen treatment revealed that only one paralog pair (CYP76A4/CYP76A6) had the similar expression pattern, suggesting functional divergence among PnCYP. Structural analysis showed that the frequency of segment duplication in $P$. notoginseng was greater than that of tandem duplication, and the $\mathrm{Ka} / \mathrm{Ks}$ ratio of two paralogous pairs (Pn-Pn) and six orthologous pairs (Pn-Pg) was greater than 1.0. All of these demonstrated that in the process of evolution, some genes undergo positive selection, and the phenomenon of functional divergence did existed.

In plants, nitrogen treatment affected some phytohormones' synthesis, such as ABA, GA, JA and SA. Transcriptome data indicated that some genes related to hormone synthesis were responsive under the three conditions. CYP707A, which is also known as ABA8Ox, was incrementally down-regulated with the three kinds of treatments, $P n C Y P 707 A 1$ showed significantly reduced expression levels with at least 2.3fold. In the qRT-PCR experiment, the PnCYP707A1 expression level was significantly decreased under ABA treatment (>2) (Fig. 7). And PnCYP707A1 promoter contained ABA relevant cis-element (ABRE). The 9-cisepoxycarotenoid dioxygenases (NCEDs) which were also associated with ABA synthesis were identified (Table S12). And PnNCED3, PnNCED4 and PnNCED5 showed sharp decrease of transcripts in at least one 


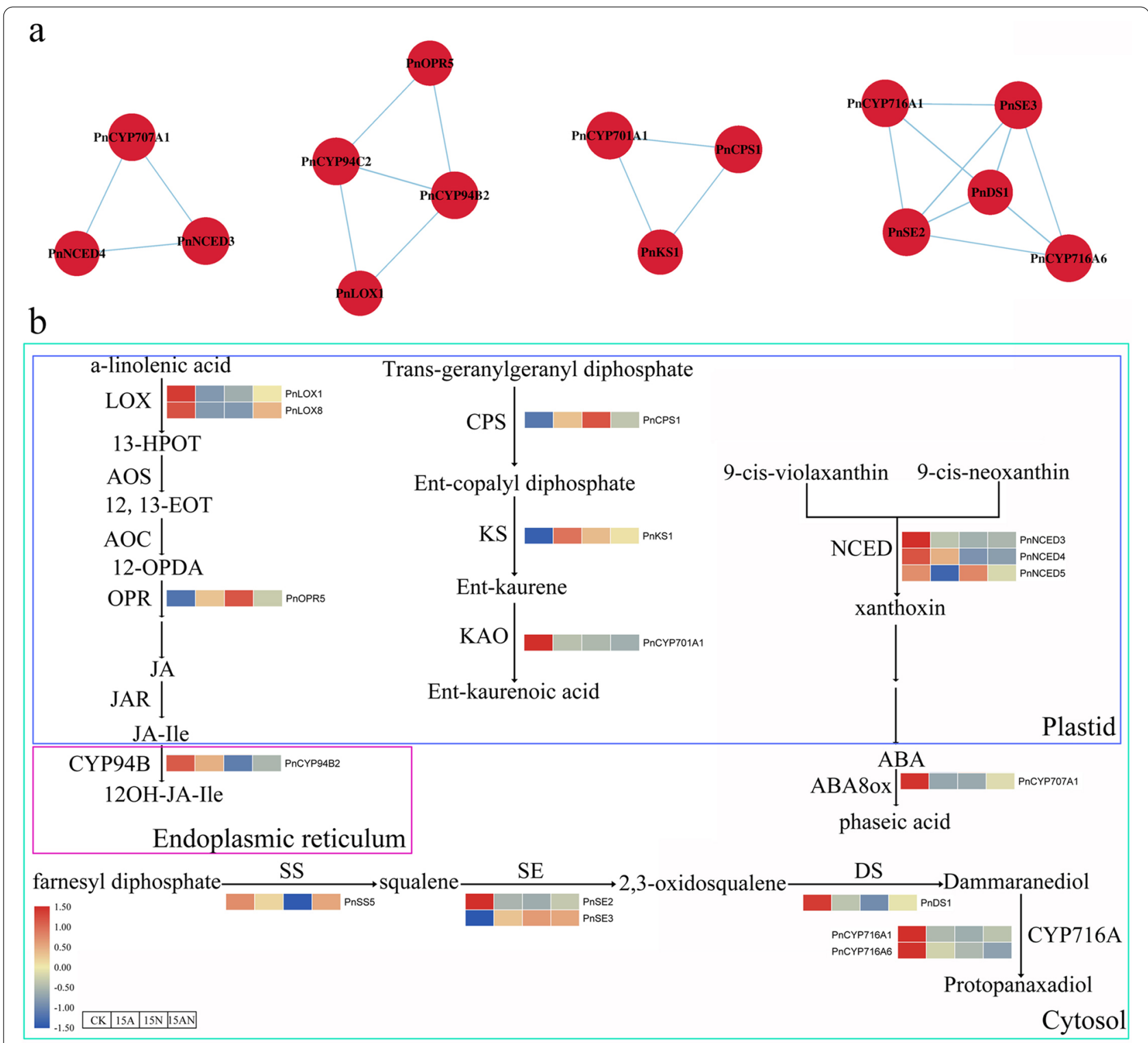

Fig. 8 Analysis of the CYP450s expression under nitrogen treatment. A: Co-expression analysis of PnCYP450s involved in ABA, JA, GA, Saponins. B: A schematic diagram of CYP450-mediated signaling pathways under nitrogen treatment

treatment. PnNCED3 was down-regulated 4.4-fold and 6.2 -fold respectively under $15 \mathrm{~A}$ and $15 \mathrm{AN}$ treatment. While PnNCED4 was significantly up-regulated in $15 \mathrm{~N}$. Furthermore, PnCYP707A1, PnNCED3 and PnNCED4 showed a significant correlation of expression (Fig. 8a). In the JA synthesis pathway, two lipoxygenases (PnLOXs) (PnLOX1 and PnLOX8) were down regulated under $15 \mathrm{~A}$ and $15 \mathrm{~N}$ treatment (Fig. 8b). Eight 12-oxophytodienoate reductases $(P n O P R s)$ were identified, however, only one of them (PnOPR8) responded to nitrogen treatment. In Arabidopsis, CYP94B1, $C Y P 94 B 3$ and $C Y P 94 C 1$ were found to be associated with the partial deactivation of JA-Ile hormone [47]. $P n C Y P 94 B 2, P n C Y P 94 C 2$ and $P n C Y P 94 C 3$ whose promoters contain the CGTCA-motif and TGACG-motif (Table S5) were all differentially expressed genes in this study. In the qRT-PCR experiment, the PnCYP94B2 expression level was significantly increased under ABA treatment $(>2)$ (Fig. 7). In co-expression network, $P n C Y P 94 B 2, P n C Y P 94 C 2$ showed a connection to PnLOX1 and PnOPR8. Moreover, GA synthesis is also affected by nitrogen in the soil [30]. Only one entcopalyl diphosphate synthase gene (CPS) was identified and it $(P n C P S 1)$ had a more than 3-fold increase in 
expression within $15 \mathrm{~N}$ treatment. Its downstream gene, kaurene synthases enzyme (PnKS1) was up-regulated 2.2 -fold when treated with 15A. And PnCYP701A1 which was also involved in gibberellin synthesis and contained the GARE-motif in its promoter, showed significant downregulation in all treatments. Likewise, the co-expression analysis indicated that these three genes were closely related to one another (Fig. 8a).

Saponins are the main medicinal ingredients of $P$. notoginseng [2], and CYP genes participate in saponin synthesis [19-23], so the genes related to the saponin synthesis pathway were identified and their transcripts levels were counted. Squalene synthase (SS) was responsible for the synthesis of triterpene essential substrate (C30 isoprenoid squalene), which showed decreased expression levels under 15A (Fig. 8b). Triterpenoid compounds oxidation genes (squalene epoxidase [SE]) $P n S E 2$ showed at least 2.9-fold down regulation under all three conditions, while PnSE3 was up-regulated at least 2.0-fold under all three conditions. Only one dammarenediol-II synthase (DS) gene, which was a kind of oxidosqualene cyclase, was found in P. notoginseng. It was down-regulated in response to $15 \mathrm{~A}$ and $15 \mathrm{~N}$. CYP716A, which plays roles in processing protopanoxadiol [23],also responded to nitrogen treatment. PnCYP716A1 and PnCYP716A6 showed significantly down-regulated expression levels in three treatments. According to the tissue expression pattern analysis, the expression levels of $P n C Y P 716 A 1$ and $P n C Y P 716 A 6$ were highest in the roots, which are the main medicinal tissue of $P$. notoginseng [2]. These two genes both responded to MEJA and ABA treatment. PnSE2, PnSE3, $P n D S 1, P n C Y P 716 A 1$ and $P n C Y P 716 A 6$ showed a significant correlation of expression (Fig. 8a). $P n C Y P$ genes participating in the synthesis of hormones and saponin pathways were showed in Fig. 8b.

\section{Conclusions}

We identified $188 \mathrm{PnCYP}$ genes, which were divided into 40 subfamilies and clustered into 9 clans. We have performed phylogeny, gene structure, chromosome location, duplicated event, GO and KEGG annotation, and functional divergence analysis on this supergene family, which showed that PnCYP genes have undergone frequent evolution and functional diversity. Through the analysis of the transcriptome under different nitrogen treatments, some genes involved in the synthesis of plant hormones such as ABA, JA and GA were identified, and some genes involved in saponin synthesis were also identified. These five genes were screened for tissue expression pattern analysis and the expression level change analysis under hormone treatment. Our findings can provide a reference for studying the functional diversification, the supergene family members correlation, and researched the $P n C Y P$ genes involved in the biosynthesis of notoginseng saponins.

\section{Methods}

\section{Identification and characterization of PnCYP450s}

The $P$. notoginseng sequences were downloaded from the Chinese Herbal Plant Genome Database (http:// www.herbal-genome.cn) [48] and CNGBdb Public website (http://ftp.cngb.org/pub/CNSA/). PnCYPs were identified according to the following procedure. First, we downloaded the CYP450 sequences of other species (A. thaliana, O. sativa) from the EnsemblPlants website (https://plants.ensembl.org/index.html) and $P$. ginseng CYPs from the National Center for Biotechnology Information (NCBI) website (https://pubmed.ncbi. nlm.nih.gov/30512034/). The Populus trichocarpa and Physcomitrella patens CYP450 protein sequences were downloaded from the P450 Homepage (http://drnel son.uthsc.edu/cytochromeP450.html) [32]. The AtCYP450s, OsCYP450s and PgCYP450s were compared with the $P$. notoginseng protein database by local blast comparison, and we searched $P$. notoginseng genes that had sequence similarities with those CYP450s. Next, Hidden Markov Model (HMM) profile in the Pfam database [49] (http://pfam.janelia.org/search/seque nce) and NCBI Conserved Domains searches (http:// www.ncbi.nlm.nih.gov/Structure/cdd/wrpsb.cgi) were peformed to see if these candidate genes contained the P450 domain (PF00067). Finally, the genes without a complete $\mathrm{P} 450$ domain were removed. The number of amino acids, open reading frame (ORF) length, isoelectric point $(\mathrm{pI})$ and molecular weight $(\mathrm{Mw})$ were estimated by ExPASy (http://www.expasy.ch/tools/pi_tool. html) [50].

\section{Phylogenetic tree construction and structure analysis of PnCYP450 gene family members}

To investigate the phylogenetic relationships among the CYPs, the CYP450 protein sequences of Physcomitrella patens (moss), O. sativa (rice), A. thaliana (Arabidopsis), P. trichocarpa (poplar), P. notoginseng and $P$. ginseng ( $P$. ginseng) were aligned by Clustalw. Neighbor-Joining method was employed to construct an un-rooted phylogenetic tree using the MEGA 5.0 software, and the replications were set as 1000 [51]. Both the phylogenetic trees of six species and individual $P$. notoginseng phylogenetic trees were constructed using the same method.

The Multiple Expectation Maximization for Motif Elicitation (MEME) online tool (http://meme.sdsc.edu/ meme/intro.html) [52] was used to identify PnCYP 
conserved motifs with the following parameters: the number of different motifs was set as as 20 , and the range of motif width were set as 6 to 50 .

Chromosome sequence information was downloaded from NCBI website with the accession number JACBWS000000000 [53]. And chromosome location map was utilized by TBtools [54].

\section{Cis-regulatory elements and miRNA targets prediction}

The $1500 \mathrm{bp}$ upstream/downstream sequences of each $P n C Y P$ gene encoding region were extracted by TBtools [54]. The sequences were then submitted and analyzed on PlantCARE website (http://bioinformatics.psb. ugent.be/webtools/plantcare/html/) to identify putative cis-elements in promoter [55]. The putative cisacting elements relative to phytohormone responses, the regulation of plant growth and development, biotic and abiotic stress responses were summarized. The $P$. notoginseng miRNA sequences were downloaded from the NCBI website (https://www.ncbi.nlm.nih.gov/pmc/ articles/PMC5573331/) [56]. Targets of miRNA were identified by psRNATarget website (http://plantgrn. noble.org/v1_psRNATarget/?function=3) [57], and the parameters were set as follow: maximum expectation $=3.0$, top target genes for each small RNA $=200$, length for complementarity scoring (hspsize) $=20$, target accessibility - allowed maximum energy to unpair the target site $(\mathrm{UPE})=25$.

\section{Selective pressure analysis}

Phylogeny-based and bidirectional best-hit methods were used to identify the homologous pair of putative paralogous pairs in $P$. notoginseng, orthologous pairs between $P$. notoginseng and $A$. thaliana, $P$. notoginseng and $P$. ginseng [58]. Nucleotide sequences were used in this analysis. The length of each gene in a homologous pair was longer than $300 \mathrm{bp}$, and the sequence similarity between two genes in each homologous pair was greater than 40\% [59]. MEGA 5.0 was used to perform genes pair alignment, and Dnasp 5.10.1 software [60] was used to calculate the synonymous substitution rate (Ks) and non-synonymous substitution rate (Ka) substitutions per site between duplicated gene pairs [61]. Values of $(\mathrm{Ka} / \mathrm{Ks})<1,=1$ and $>1$ indicate negative selection, neutral evolution and positive selection, respectively [62].

\section{GO, KEGG annotation analysis and subcellular localization prediction}

The 188 PnCYP450 protein sequences were annotated using the Blast2GO software $[63,64]$. The annotation results were obtained with following parameters: e-value was $1.0 \mathrm{E}-6$, annotation cutoff was 55 , Go weight was 5 . KAAS website (https://www.genome.jp/kaas-bin/kaas main) and BlastKOALA website (https://www.kegg.jp/ blastkoala/) were used to perform KEGG annotation [65]. On the KAAS website, the BBH (bi-directional best hit) assignment method was used. And A.thaliana, P. trichocarpa and Vitis vinifera were selected as GENES data set. The family_eukaryotes genus_prokaryotes database was used on the BlastKOALA website. The subcellular localization of the PnCYP proteins was predicted using CELLO v.2.5 (http://cello.life.nctu.edu.tw/) [66].

\section{Estimation of functional divergence}

The DIVERGE 3.0 software was used to study whether functional divergence occurred among the CYPs subfamily members [67]. There were two main types of functional divergence: Type I represented the evolutionary rate change between replicated genes, where a site that was conserved in one gene but changed in another gene, and Type II represented the evolutionary nature change of replicated genes $[68,69]$. If the correlation coefficients $\left(\theta_{\mathrm{I}}\right.$ and $\theta_{\text {II }}$ ) were significantly greater than 0 , it means that functional divergence has occurred. We then calculated posterior probability value $\left(\mathrm{Q}_{\mathrm{k}}\right)$, and when a site with a $\mathrm{Q}_{\mathrm{k}}$ value greater than 0.67 , it can be defined as the functional divergence-related site. SPSS software was used for a t-value test to analyze the significant difference of the posterior probability value, and the significance threshold set as ${ }^{*} P<0.05$.

\section{Expression and co-expression network and analysis of PnCYP450 genes treated with different forms of nitrogen fertilizers}

To study the expression levels of PnCYP450 genes when $P$. notoginseng roots were treated with different forms of nitrogen fertilizers $\left(15 \mathrm{mM} \mathrm{NH}{ }^{4+}[15 \mathrm{~A}], 15 \mathrm{mM} \mathrm{NO}^{3-}\right.$ $[15 \mathrm{~N}], 15 \mathrm{mM} \mathrm{NH}^{4+}+15 \mathrm{mM} \mathrm{NO}^{3-}$ [15AN]), the RNAseq data were downloaded from NCBI GEO DataSet (https://www.ncbi.nlm.nih.gov/gds/) with accession number GSE112437 and NCBI Sequence Read Archive (SRA) database (https://www.ncbi.nlm.nih.gov/sra) with accession number SRP136626. Genes with fold change (FC) greater than 1.5 and significant $p$-values $<0.05$ were defined as differentially expressed genes [70]. The FPKM values of $P_{n} C Y P s$ and other genes which participated in hormone synthesis were used for heat map generation by TBtools software. And scale methods were set as a row scale in the heat map drawing. Co-expression networks were performed using the OmicStudio tools (https:// www.omicstudio.cn/index), and $p$-values $<0.05$ were considered statistically significant.

\section{Plant material and real-time PCR analysis}

Growing two-year-old $P$. notoginseng was collected from the Wenshan County, Yunnan Province, China. For plant hormone treatment, $P$. notoginseng leaves were treated with $10 \mu \mathrm{M}$ ABA and $100 \mu \mathrm{M}$ MEJA. These 
treated $P$. notoginseng plants were grown in greenhouses under $14 / 10 \mathrm{~h}$ of light/dark conditions at a continuous temperature of $23 \pm 1{ }^{\circ} \mathrm{C}$. Total RNA of untreated plant roots, stems, leaves flowers and hormone-treated leaves were extracted using Trizol (Sangon Biotech, Shanghai, China) according to the manufacturer's instructions. First-strand cDNA was synthesized by a Prime-Script ${ }^{\mathrm{TM}}$ RT Reagent Kit (TaKaRa, Tokyo, Japan) according to the manufacturer's instructions. Primer Premier 5.0 was used to design the specific primers (Table S13) required in real-time PCR experiments, 26S RNA was used as an internal reference [71]. In qRT-PCR, $20 \mu \mathrm{L}$ reaction volume contained $10 \mu \mathrm{L} 2 \times$ ApexHF FS PCR Master Mix, $0.4 \mu \mathrm{L}$ forward primer, $0.4 \mu \mathrm{L}$ reverse primer, $1 \mu \mathrm{L}$ cDNA template and $8.2 \mu \mathrm{L}$ ddH2O. qPCR reaction program was set as follows: $98^{\circ} \mathrm{C}$ for $10 \mathrm{~s}$; 35 cycles of $55^{\circ} \mathrm{C}$ for $10 \mathrm{~s}$ and $72^{\circ} \mathrm{C}$ for $10 \mathrm{~s}$. GraphPad 8.0 software was used for data analysis.

\begin{abstract}
Abbreviations
CYP: Cytochrome P450; GO: Gene Ontology; P. notoginseng: Panax notoginseng; AOS: Allene oxide synthase; JA: Jasmonate; ABA: Abscisic acid; ABA8ox: ABA 8'-hydroxylases; GA: Gibberellin; KO: Ent-kaurene oxidase; KAO: Ent-kaurenoic acid oxidase; BR: Brassinosteroid; HMM: Hidden Markov Model; ORF: Open reading frame; pl: Isoelectric point; Mw: Molecular weight; Arabidopsis: Arabidopsis thaliana; rice: Oryza sativa; moss: Physcomitrella patens; poplar: Populus trichocarpa; P. ginseng: Panax ginseng; MEME: Multiple Expectation Maximization for Motif Elicitation; UPE: Unpair the target site; Ks: Synonymous substitution rate; Ka: Non-synonymous substitution rate; $Q_{k}$ : Posterior probability value; $15 \mathrm{~A}: 15 \mathrm{mM} \mathrm{NH}^{4+}$; $15 \mathrm{~N}^{2} 15 \mathrm{mM} \mathrm{NO}^{3-}$; $15 \mathrm{AN}: 15 \mathrm{mM}$ $\mathrm{NH}^{4+}+15 \mathrm{mM} \mathrm{NO}^{3-}$; SRA: Sequence Read Archive; FC: Fold change; FPKM: Per Million mapped reads; SA: Salicylic acid; SS: Squalene synthase; SE: Squalene epoxidase; DS: Dammarenediol-II synthase.
\end{abstract}

\section{Supplementary Information}

The online version contains supplementary material available at https://doi. org/10.1186/s12870-021-03224-x.

Additional file 1: Figure S1. Phylogeny of CYP450s from Panax notoginseng, Arabidopsis thaliana, Panax ginseng, Oryza sativa, Populus trichocarpa and Physcomitrella patens.

Additional file 2: Figure S2. Distribution of Ka and Ks from paralogous $(\mathrm{Pn}-\mathrm{Pn})$ and orthologous (Pn-Pg and $\mathrm{Pn}$-At) gene pairs. Different shapes and colors represented homologous gene pairs of $\mathrm{Pn}-\mathrm{Pn}, \mathrm{Pn}-\mathrm{Pg}$ and $\mathrm{Pn}-\mathrm{At}$, respectively, and the black line indicates that the slope of $\mathrm{Ka} / \mathrm{Ks}=1$.

Additional file 3: Figure S3. Evolutionary and gene structure analysis of PnCYP genes. Schematic representation of 20 conserved motifs in PnCYP genes. Conserved motifs in PnCYP genes were identified using MEME. Different colored boxes represent different motifs. Box lengths in the figure do not represent actual relative motif sizes. Exons and introns are indicated by yellow rectangles and gray lines, respectively.

Additional file 4: Figure S4. Differential expression of PnCYP genes under nitrogen fertilizers treatment. 15A:15 $\mathrm{mM} \mathrm{NH}^{4+}, 15 \mathrm{~N}: 15 \mathrm{mM} \mathrm{NO}^{3-}$, 15AN: $15 \mathrm{mM} \mathrm{NH}^{4+}+15 \mathrm{mM} \mathrm{NO}^{3-}$

Additional file 5: Table S1. Detailed information about the PnCYPs in the Panax notoginseng.

Additional file 6: Table S2. Paralogous (Pn-Pn) and orthologous (Pn-Pg and $\mathrm{Pn}$-At) gene pairs.
Additional file 7: Table S3. Ka, Ks, Ka/Ks values for the PnCYPs genes in Panax notoginseng.

Additional file 8: Table S4. Ka, Ks, Ka/Ks values for the PnCYPs genes in Panax notoginseng, Panax ginseng, and Arabidosis.

Additional file 9: Table S5. Number of each cis-acting element in the promoter regions of PnCYPs genes.

Additional file 10: Table S6. List of predicted miRNA-target interactions.

Additional file 11: Table S7. Detailed information list of GO annotation and subcellular localization prediction of PnCYP proteins.

Additional file 12: Table S8. List of CYP51, CYP74, CYP97 members functional divergence results.

Additional file 13: Table S9. List of FKPM values of 187 PnCYP450s under three kinds of nitrogen fertilizers treatment.

Additional file 14: Table S10. List of 107 PnCYP genes whose expression data $($ FPKM) $>1$ in one or more treatment.

Additional file 15: Table S11. List of 47 PnCYP differentially expressed genes.

Additional file 16: Table S12. List of identified P. notoginseng ABA, JA, $\mathrm{GA}$, saponins biosynthesis related genes.

Additional file 17: Table S13. Primers used for the GRT-PCR analysis of PnCYP gene expression.

\section{Acknowledgments}

We are grateful to the providers who submitted the RNA-seq data to the public expression databases which can be applied freely.

\section{Authors' contributions}

RX conceived the study, put into effect the main bioinformatics analyses, and drafted the manuscript. TH carried out the software analyses and helped to construct the figures and Tables. YW took part in the experiments and drafting of the manuscript. SL and YG processed the experimental data and helped to draft the manuscript. HY reviewed the project and helped in revamping the manuscript. YX conceived and guided the experiments, and helped in coordinating the project and drafting the manuscript. All authors read and accepted the final manuscript. The author(s) read and approved the final manuscript.

\section{Funding}

This study was supported by the National Science and Technology Support Program (Grant No. 2015BAD07B070104) and National Natural Science Foundation of China (Grant No. 31670672). The funders have no role in the design of the study and collection, analysis, and interpretation of data and in writing the manuscript.

\section{Availability of data and materials}

All the supporting data are included within the article and its additional files.

\section{Declarations}

Ethics approval and consent to participate

Not applicable.

\section{Consent for publication}

Not applicable.

\section{Competing interests}

The authors declare that they have no competing interests.

\section{Author details}

${ }^{1}$ Laboratory of Modern Biotechnology, School of Forestry and Landscape Architecture, Anhui Agricultural University, Hefei 230036, China. ${ }^{2}$ National Engineering Laboratory of Crop Stress Resistance Breeding, Anhui Agricultural University, Hefei 230036, China. 
Received: 12 June 2021 Accepted: 23 September 2021

Published online: 06 October 2021

\section{References}

1. Wang T, Guo RX, Zhou GH, Zhou XD, Kou ZZ, Sui F, et al. Traditional uses, botany, phytochemistry, pharmacology and toxicology of Panax notoginseng (Burk.) FH Chen: a review. J Ethnopharmacol. 2016;188:234-58.

2. Men SY, Huo QL, Shi L, Yan Y, Yang CC, Yu W, et al. Panax notoginseng saponins promotes cutaneous wound healing and suppresses scar formation in mice. J Cosmet Dermatol. 2020;19(2):529-34.

3. Dan M, Su MM, Gao XF, Zhao T, Zhao AH, Xie GX, et al. Metabolite profiling of Panax notoginseng using UPLC-ESI-MS. Phytochemistry. 2008;69(11):2237-44

4. Sun HX, Yang ZG, Ye YP. Structure and biological activity of protopanaxatriol-type saponins from the roots of Panax notoginseng. Int Immunopharmacol. 2006:6:14-25.

5. Ng TB. Pharmacological activity of Sanchi-ginseng (Panax notoginseng). J Pharm Pharmacol. 2006:58:1007-19.

6. Konoshima T, Takasaki M, Tokuda $\mathrm{H}$. Anti-carcinogenic activity of the roots of Panax notoginseng II. Biol Pharm Bull. 1999:22:1150-2.

7. Mizutani M, Ohta D. Masaharu Mizutani and Daisaku Ohta. Diversification of P450 genes during land plant evolution. Annu Rev Plant Biol. 2010;61:291-315

8. Ai JW, Zhu Y, Duan J, Yu Q, Zhang G, Wan F, Xiang ZH. Genome-wide analysis of cytochrome P450 monooxygenase genes in the silkworm, Bombyx mori. Gene. 2011;480:0-50.

9. Nelson D, Werck-Reichhart D. A P450-centric view of plant evolution. Plant J. 2011;66(1):194-211.

10. Dobritsa AA, Shrestha J, Morant M, Pinot F, Matsuno M, Swanson R, et al. CYP704B1 is along-chain fatty acid omega-hydroxylase essential for sporopollenin synthesis in pollen of Arabidopsis. Plant Physiol. 2009;151(2):574-89.

11. Kandel S, Sauveplane V, Olry A, Diss L, Benveniste I, Pinot F. Cytochrome P450-dependent fatty acid hydroxylases in plants. Phytochem Rev. 2006;5(2-3):359-72.

12. Morant M, Jorgensen $K$, Schaller $H$, Pinot F, Moller BL, Werck-Reichhart D, et al. CYP703 is an ancient cytochrome P450 in land plants catalyzing in-chain hydroxylation of Lauric acid to provide building blocks for Sporopollenin synthesis in pollen. Plant Cell. 2007;19(5):1473-87.

13. Wasternack C, Feussner I. The Oxylipin pathways: biochemistry and function. Annu Rev Plant Biol. 2018;69(1):363.

14. Kim J, Smith JJ, Tian L, DellaPenna D. The evolution and function of carotenoid hydroxylases in Arabidopsis. Plant Cell Physiol. 2009;50(3):463-79.

15. Morrone D, Chen XM, Coates RM, Peters RJ. Characterization of the kaurene oxidase CYP701A3, a multifunctional cytochrome P450 from gibberellin biosynthesis. Biochem J. 2010;431(3):337-44.

16. Regnault T, Daviere JM, Heintz D, Lange T, Achard P. The gibberellin biosynthetic genes AtKAO1 and AtKAO2 have overlapping roles throughout Arabidopsis development. Plant J Cell Mol Biol. 2014;80(3):462-74.

17. Pan ZQ, Baerson SR, Wang M, Bajsa-Hirschel J, Rimando AM, Wang XQ, et al. A cytochrome P450 CYP71 enzyme expressed in Sorghum bicolor root hair cells participates in the biosynthesis of the benzoquinone allelochemical sorgoleone. New Phytol. 2018:218(2):616-29.

18. Pakdeechanuan P, Teoh S, Shoji T, Hashimoto T. Non-functionalization of two CYP82E nicotine N-Demethylase genes abolishes Nornicotine formation in Nicotiana langsdorffii. Plant Cell Physiol. 2012;53(12):2038-46.

19. Geisler K, Hughes RK, Sainsbury F, Lomonossoff GP, Rejzek M, Fairhurst S, et al. Biochemical analysis of a multifunctional cytochrome P450 (CYP51) enzyme required for synthesis of antimicrobial triterpenes in plants. $P$ Natl Acad Sci USA. 2013;110(35):E3360-7.

20. Shibuya M, Hoshino M, Katsube Y, Hayashi H, Kushiro T, Ebizuka Y. Identification of beta-amyrin and sophoradiol 24-hydroxylase by expressed sequence tag mining and functional expression assay. FEBS J. 2006;273(5):948-59.

21. Seki H, Ohyama K, Sawai S, Mizutani M, Ohnishi T, Sudo H, et al. Licorice $\beta$-amyrin 11-oxidase, a cytochrome P450 with a key role in the biosynthesis of the triterpene sweetener glycyrrhizin. P Natl Acad Sci USA. 2008;105:14204-9.
22. Seki H, Sawai S, Ohyama K, Mizutani M, Ohnishi T, Sudo H, et al. Triterpene Functional Genomics in Licorice for Identification of CYP72A154 Involved in the Biosynthesis of Glycyrrhizin. Plant Cell. 2011;23(11):4112-23.

23. Carelli M, Biazzi E, Panara F, Tava A, Scaramelli L, Porceddu A, et al. Medicago truncatula CYP716A12 is a multifunctional oxidase involved in the biosynthesis of hemolytic Saponins. Plant Cell. 2011;23(8):3070-81.

24. Tamiru M, Undan JR, Takagi H, Abe A, Yoshida K, Undan JQ et al. A cytochrome P450, OsDSS1, is involved in growth and drought stress responses in Rice (Oryza sativa L.). Plant Mol Biol. 2015;88:85-99.

25. Mao GH, Seebeck T, Schrenker D, Yu O. CYP709B3, a cytochrome P450 monooxygenase gene involved in salt tolerance in Arabidopsis thaliana. BMC Plant Biol. 2013;13:169.

26. Liu Q, Khakimov B, Cardenas PD, Cozzi F, Olsen CE, Jensen KR, et al. The cytochrome P450 CYP72A552 is key to production of hederagenin-based saponins that mediate plant defense against herbivores. New Phytol. 2019;222(3):1599-609.

27. Rustgi S, Springer A, Kang C, von Wettstein D, Reinbothe C, Reinbothe S, Pollmann S. ALLENE OXIDE SYNTHASE and HYDROPEROXIDE LYASE, two non-canonical cytochrome P450s in Arabidopsis thaliana and their different roles in plant defense. Int J Mol Sci. 2019:20(12):3064.

28. LiWQ, Shao M, Yang J, Zhong WG, Okada K, Yamane H, et al. Oscyp71Z2 involves diterpenoid phytoalexin biosynthesis that contributes to bacterial blight resistance in rice. Plant Sci. 2013;207:98-107.

29. Robert PD, Douglas GL. Multiple forms of plant cytochromes P450. Plant Physiol. 1991;96:669-74.

30. Luo J, Zhou J, Li H, Shi WG, Polle A, Lu MZ, et al. Global poplar root and leaf transcriptomes reveal links between growth and stress responses under nitrogen starvation and excess. Tree Physiol. 2015;35(12):1283-302.

31. Paquette $S M, B a k S$, Feyereisen R. Intron-exon organization and phylogeny in a large superfamily, the paralogous cytochrome P450 genes of Arabidopsis thaliana. DNA Cell Biol. 2000;19:307-17.

32. Wei K, Chen H. Global identification, structural analysis and expression characterization of cytochrome P450 monooxygenase superfamily in rice. BMC Genomics. 2018;19:35.

33. Jiu ST, Xu Y, Wang JY, Wang L, Liu XJ, Sun WX, et al. The cytochrome P450 Monooxygenase inventory of grapevine (Vitis vinifera L.): genome-wide identification, evolutionary characterization and expression analysis analysis. Front Genet. 2020;11:44.

34. Wang $Y, L i X Y$, Lin YP, Wang YF, Wang KY, Sun CY, et al. Structural variation, functional differentiation, and activity correlation of the cytochrome P450 gene superfamily revealed in ginseng. Plant Genome. 2018;11(3):170106.

35. Xia PG, Guo HB, Zhao HG, Jiao J, Deyholos MK, Yan XJ, et al. Optimal fertilizer application for panax notoginseng and effect of soil water on root rot disease and saponin contents. J Ginseng Res. 2016;40(1):38-46.

36. Wei W, Yang M, Liu YX, Huang HC, Ye C, Zheng JF, et al. Fertilizer $n$ application rate impacts plant-soil feedback in a sanqi production system. Sci Total Environ. 2018:633:796-807.

37. Kiba T, Kudo T, Kojima M, Sakakibara H. Hormonal control of nitrogen acquisition: roles of auxin, abscisic acid, and cytokinin. J Exp Bot. 2011;62:1399-409.

38. Vidal EA, Moyano TC, Riveras E, Contreras-Lopez O, Gutierrez RA. Systems approaches map regulatory networks downstream of the auxin receptor AFB3 in the nitrate response of Arabidopsis thaliana roots. Proc Natl Acad Sci U S A. 2013;110(31):12840-5.

39. Li YX, Wei KF. Comparative functional genomics analysis of cytochrome P450 gene superfamily in wheat and maize. BMC Plant Biol. 2020;20(1):93.

40. Sunkar R, Zhou X, Yun Z, Zhang W, Zhu JK. Identification of novel and candidate miRNAs in Rice by high throughput sequencing. BMC Plant Biol. 2008;8(1):25

41. Mohanta TK, Khan A, Hashem A, Abd Allah EF, Al-Harrasi A. The molecular mass and isoelectric point of plant proteomes. BMC Genomics. 2019;20:631.

42. Hamberger B, Bak S. Plant P450s as versatile drivers for evolution of species-specific chemical diversity. Philos TR Soc B. 2013;368(1612):20120426.

43. Ohnishi T, Watanabe B, Sakata K, Mizutani M. CYP724B2 and CYP90B3 function in the early C-22 hydroxylation steps of Brassinosteroid biosynthetic pathway in tomato. J Agric Chem Soc Japan. 2006;70(9):2071-80.

44. Ohnishi T, Godza B, Watanabe B, Fujioka S, Hategan L, Ide K, et al. CYP90A1/CPD, a brassinosteroid biosynthetic cytochrome P450 of Arabidopsis, catalyzes C-3 oxidation. J Biol Chem. 2012;287:31551-60. 
45. Takei K, Yamaya T, Sakakibara H. Arabidopsis CYP735A1 and CYP735A2 encode Cytokinin hydroxylases that catalyze the biosynthesis of transZeatin. J Biol Chem. 2004;279(40):41866-72.

46. Heitz T, Widemann E, Lugan R, Miesch L, Ullmann P, Desaubry L, et al. Cytochromes P450 CYP94C1 and CYP94B3 catalyze two successive oxidation steps of plant hormone Jasmonoyl-isoleucine for catabolic turnover. J Biol Chem. 2012;287(9):6296-306.

47. Li H, Pinot F, Sauveplane V, Werck-Reichhart D, Diehl P, Schreiber L, et al. Cytochrome P450 family member CYP704B2 catalyzes the omegahydroxylation of fatty acids and is required for anther Cutin biosynthesis and pollen Exine formation in Rice. Plant Cell. 2010;22(1):173-90.

48. Chen W, Kui L, Zhang GH, Zhu SS, Zhang J, Wang X, et al. Whole-genome sequencing and analysis of the Chinese herbal plant Panax notoginseng. Mol Plant. 2017;10(6):899-902.

49. Bateman A, Coin L, Durbin R, Finn RD, Hollich V, Griffiths-Jones S, et al. The Pfam protein families database. Nucleic Acids Res. 2000;28:263-6.

50. He HS, Dong Q, Shao YH, Jiang HY, Zhu SW, Cheng BJ, et al. Genomewide survey and characterization of the WRKY gene family in Populus trichocarpa. Plant Cell Rep. 2012;31:1199-217.

51. Tamura K, Peterson D, Peterson N, Kumar S. MEGA5: molecular evolutionary genetics analysis using maximum likelihood, evolutionary distance, and maximum parsimony methods. Mol Biol Evol. 2011;28:2731-9.

52. Bailey TL, Boden M, Buske FA, Frith M, Grant CE, Clementi L, et al. MEME SUITE: tools for motif discovery and searching. Nucleic Acids Res. 2009;37:W202-8.

53. Fan GY, Liu XC, Sun S, Shi CC, Du X, Han K, et al. The chromosome level genome and genome-wide association study for the agronomic traits of Panax Notoginseng. ISCIENCE. 2020;23(9):101538.

54. Chen CJ, Chen H, Zhang Y, Thomas HR, Frank MH, He YH, et al. TBtools: an integrative toolkit developed for interactive analyses of big biological data. Mol Plant. 2020;18(8):1194-202.

55. Liu H, Wang $X$, Zhang H, Yang Y, Ge X, Song F. A rice serine carboxypeptidase-like gene OsBISCPL1 is involved in regulation of defense responses against biotic and oxidative stress. Gene. 2008;420:57-65.

56. Zheng Y, Chen $K, X u$ ZN, Liao PR, Zhang XT, Liu L, et al. Small RNA profiles from Panax notoginseng roots differing in sizes reveal correlation between miR156 abundances and root biomass levels. Sci Rep. 2017;7:9418.

57. Dai XB, Zhao PX. psRNATarget: a plant small RNA target analysis server. Nucleic Acids Res. 2011;39:W155-9.
58. Altschul SF, Madden TL, Schäffer AA, Zhang J, Zhang Z, Miller W, et al. Gapped BLAST and PSI-BLAST: a new generation of protein database search programs. Nucleic Acids Res. 1997;25(17):3389-402.

59. Wang YJ, Liu HL, Zhu DY, Gao YM, Yan HW, Xiang Y. Genome-wide analysis of VQ motif-containing proteins in Moso bamboo (Phyllostachys edulis). Planta. 2017;246(1):165-81.

60. Librado P, Rozas J. DnaSP $\vee 5$ : a software for comprehensive analysis of DNA polymorphism data. Bioinformatics. 2009;25(11):1451-2.

61. Hu RB, Qi GA, Kong YZ, Kong DJ, Gao QA, Zhou GK. Comprehensive analysis of NAC domain transcription factor gene family in Populus trichocarpa. BMC Plant Biol. 2010;10(10):1-23.

62. Hurst LD. The Ka/Ks ratio: diagnosing the form of sequence evolution. Trends Genet. 2002;18:486.

63. Ashburner M, Ball CA, Blake JA, Botstein D, Butler H, Cherry JM, et al. Gene ontology: tool for the unification of biology. Nat Genet. 2000;25:25-9.

64. Conesa A, Gotz S. Blast2GO: a comprehensive Suite for functional analysis in plant genomics. Int J Plant Prod. 2008;2008:619832.

65. Kanehisa M, Sato Y, Morishima K. BlastKOALA and GhostKOALA: KEGG tools for functional characterization of genome and metagenome sequences. J Mol Biol. 2016;428:726-31.

66. Yu CS, Lin CJ, Hwang JK. Predicting subcellular localization of proteins for gram-negative bacteria by support vector machines based on $n$-peptide compositions. Protein Sci. 2004;13:1402-6.

67. Gu X, Zou YY, Su ZX, Huang W, Zhou Z, Arendsee Z, et al. An update of DIVERGE software for functional divergence analysis of protein family. Mol Biol Evol. 2013;30(7):1713-9.

68. Gu X. Functional divergence in protein (family) sequence evolution. Genetica. 2003;118(2-3):133-41.

69. Gu X. A simple statistical method for estimating type-II (cluster-specific) functional divergence of protein sequences. Mol Biol Evol. 2006;23(10):1937-45.

70. Ou XH, Li SP, Liao PR, Cui XM, Zheng BL, Yang Y, et al. The transcriptome variations of Panax notoginseng roots treated with different forms of nitrogen fertilizers. BMC Genomics. 2019;20:9.

71. Wu Q, Ma XY, Zhang KF, Feng XH. Identification of reference genes for tissue-specific gene expression in Panax notoginseng using quantitative real-time PCR. Biotechnol Lett. 2015:37:197-204.

\section{Publisher's Note}

Springer Nature remains neutral with regard to jurisdictional claims in published maps and institutional affiliations.
Ready to submit your research? Choose BMC and benefit from:

- fast, convenient online submission

- thorough peer review by experienced researchers in your field

- rapid publication on acceptance

- support for research data, including large and complex data types

- gold Open Access which fosters wider collaboration and increased citations

- maximum visibility for your research: over $100 \mathrm{M}$ website views per year

At BMC, research is always in progress.

Learn more biomedcentral.com/submissions 